\title{
Towards a Gordon form of the Kerr spacetime
}

\author{
Stefano Liberati, ${ }^{1,2}$ Giovanni Tricella, ${ }^{1,2}$ and Matt Visser ${ }^{3}$ \\ ${ }^{1}$ SISSA - International School for Advanced Studies, via Bonomea 265, \\ 34136 Trieste, Italy. \\ ${ }^{2}$ INFN sezione di Trieste, via Valerio 2, Trieste, Italy. \\ ${ }^{3}$ School of Mathematics and Statistics, Victoria University of Wellington, \\ PO Box 600, Wellington 6140, New Zealand.
}

E-mail: liberati@sissa.it, gtricell@sissa.it, matt.visser@sms.vuw.ac.nz

11 March 2018; LATEX-ed 13 March 2018

\begin{abstract}
It is not currently known how to put the Kerr spacetime metric into the so-called Gordon form, although the closely related Kerr-Schild form of the Kerr metric is well known. A Gordon form for the Kerr geometry, if it could be found, would be particularly useful in developing analogue models for the Kerr spacetime, since the Gordon form is explicitly given in terms of the 4-velocity and "refractive index" of an effective medium. In the current article we report progress toward this goal. First we present the Gordon form for an approximation to Kerr spacetime in the slowrotation limit, obtained by suitably modifying the well-known Lense-Thirring form of the slow-rotation metric. Second we present the Gordon form for the Kerr spacetime in the near-null limit, (the 4-velocity of the medium being close to null). That these two perturbative approximations to the Kerr spacetime in Gordon form exist gives us some confidence that ultimately one might be able to write the exact Kerr spacetime in this form.
\end{abstract}

KeYwords: Kerr spacetime; Kerr-Schild metric; Gordon metric; Doran metric; coordinate transformation.

PACS numbers: 04.20.-q; 04.20.Jb; 04.70.-s; 04.70.Bw 


\section{Contents}

1 Introduction $\quad 2$

2 Two easy results

2.1 Gordon form of Schwarzschild spacetime . . . . . . . . . . . . 3

2.2 Gordon form of Lense-Thirring slow-rotation spacetime . . . . . . . . . . 4

3 General algorithm

3.1 Non-normalized Gordon and Kerr-Schild forms . . . . . . . . . . . . 5

3.2 How to find analytic expressions for Gordon and Kerr-Schild forms . . . 6

3.3 Checking Schwarzschild in Kerr-Schild form . . . . . . . . . . . . . . . 7

3.4 Checking Schwarzschild in Gordon form . . . . . . . . . . . . . . . . . . 8

4 The Kerr spacetime 9

4.1 Slow rotation . . . . . . . . . . . . . . . . . 10

4.1.1 General case of slow-rotating Kerr in Gordon form . . . . . . . . 11

4.1.2 Consistency check with Lense-Thirring . . . . . . . . . . . 13

4.2 Near-null Gordon form of Kerr spacetime . . . . . . . . . . . . . . . . . . 14

4.2.1 Infinitesimal local translation of the Kerr metric . . . . . . . . . 14

4.2.2 Solution in modified components . . . . . . . . . . . 15

5 Vorticity and applications to analogue spacetimes $\quad 17$

6 Further developments - towards a full solution 18

\section{Introduction}

What is now called the "Gordon form" for a general class of spacetime metrics currently dates back some 95 years — to 1923 — originally being developed as a model for studying the propagation of light in a relativistic medium [1. A spacetime metric is said to be in Gordon form if:

$$
g_{\mu \nu}=\eta_{\mu \nu}+\left(1-c_{*}^{2}\right) V_{\mu} V_{\nu} .
$$

Here $\eta_{\mu \nu}$ is some background metric (typically taken to be flat Minkowski space), while $V_{\mu}$ is some 4-velocity (properly normalized to $\eta^{\mu \nu} V_{\mu} V_{\nu}=-1$ in terms of the background metric), and $c_{*}$ can be interpreted as the speed of light in the medium (so in terms of the refractive index $c_{*}=1 / n$ ). In situations discussed below (where we might not necessarily want to adopt the moving medium interpretation) $c_{*}$ can still be interpreted as the coordinate speed of light at spatial infinity. This Gordon form for the spacetime metric has much deeper implications and a significantly wider range of applicability than 
the original context in which it was developed [2, 3, 4, 5, 6, 17, 8, 9, 10, 11, 12, 13], though only relatively recently (2004) has it become clear that the theoretically important Schwarzschild spacetime can be put into this Gordon form [14, 15].

In counterpoint, the astrophysically and observationally more important Kerr spacetime (discovered in 1963 [16]) continues to attract considerable interest and provide unexpected new discoveries [16, 17, 18, 19, 20, 21]. One specific and relatively simple form of the Kerr metric is this

$$
\begin{gathered}
\mathrm{d} s^{2}=-\mathrm{d} t^{2}+\mathrm{d} r^{2}+2 a \sin ^{2} \theta \mathrm{d} r \mathrm{~d} \phi+\left(r^{2}+a^{2} \cos ^{2} \theta\right) \mathrm{d} \theta^{2}+\left(r^{2}+a^{2}\right) \sin ^{2} \theta \mathrm{d} \phi^{2} \\
+\frac{2 m r}{r^{2}+a^{2} \cos ^{2} \theta}\left(-\mathrm{d} t \mp \mathrm{d} r \mp a \sin ^{2} \theta \mathrm{d} \phi\right)^{2} .
\end{gathered}
$$

Here $a=J / m$ is as usual the angular momentum per unit mass of the black hole. Several other forms for the Kerr metric are known [17, 18, 19]. Indeed, only relatively recently (2000) was the Doran form of the Kerr spacetime developed [20, 21]; this seeming to be as close as one can get to putting the Kerr metric into Painleve-Gullstrand "acoustic geometry" form. Now, historically it has been found that every significantly new form of the Kerr solution has lead to advances in our understanding, and it is still possible (though maybe not entirely likely) that the Kerr solution could be greatly simplified by writing it in some particularly clean form [18].

This raises the natural question - is it possible to put the Kerr spacetime into Gordon form? One reason for being particularly interested in this question is the observation that many analogue spacetimes are quite naturally presented in Gordon form. (See particularly [12, 22], or more generally [23, 24, 25] and [26, 27, 28, 29, 30, 31].) We shall partially answer this question by presenting two perturbative calculations. First we shall perform a slow-rotation calculation putting the well-known Lense-Thirring metric into Gordon form. Second we shall present a "near-null" version of Kerr in Gordon form; by starting with Kerr in Kerr-Schild form and performing an appropriate infinitesimal coordinate transformation. In view of the black hole uniqueness theorems we know that the Kerr family is unique; we are not looking for a new spacetime. Instead we are looking for a new way of writing the quite standard Kerr spacetime; we are trying to find a coordinate transformation to simplify the presentation of the Kerr spacetime.

\section{Two easy results}

To set the stage, let us first present two simple results, before developing a general algorithm for implementing infinitesimal coordinate changes.

\subsection{Gordon form of Schwarzschild spacetime}

The Gordon form of the Schwarzschild metric [14, 15] is less well-known than perhaps is should be. Consider the line element

$$
\mathrm{d} s^{2}=\left(\eta_{\mu \nu}+\left(1-c_{*}^{2}\right) V_{\mu} V_{\nu}\right) \mathrm{d} x^{\mu} \mathrm{d} x^{\nu} ; \quad V=-\sqrt{1+\frac{2 \tilde{m}}{r}} \mathrm{~d} t+\sqrt{\frac{2 \tilde{m}}{r}} \mathrm{~d} r .
$$


In spherical coordinates this is

$\mathrm{d} s^{2}=-\mathrm{d} t^{2}+\mathrm{d} r^{2}+r^{2}\left(\mathrm{~d} \theta^{2}+\sin ^{2} \theta \mathrm{d} \phi^{2}\right)+\left(1-c_{*}^{2}\right)\left(-\sqrt{1+\frac{2 \tilde{m}}{r}} \mathrm{~d} t+\sqrt{\frac{2 \tilde{m}}{r}} \mathrm{~d} r\right)^{2}$.

This is spherically symmetric and easily checked to be Ricci flat — so by Birkhoff's theorem it must be Schwarzschild spacetime in disguise. Here $c_{*}$ is an arbitrary constant $c_{*} \in(0,1)$, which at spatial infinity can be viewed, as anticipated, as the coordinate speed of light. Furthermore $V_{a}$ is a 4-velocity, (normalized in the background metric, $\eta_{\mu \nu} V^{\mu} V^{\nu}=-1$ ), and the parameter $\tilde{m}$ is proportional to the physical mass of the Schwarzschild spacetime. By noting that

$$
g_{t t}=-1+\left(1-c_{*}^{2}\right)(1+2 \tilde{m} / r)=-c_{*}^{2}+\left(1-c_{*}^{2}\right) 2 \tilde{m} / r,
$$

and comparing to the asymptotic behaviour of Schwarzschild in the usual curvature coordinates, we identify the physical mass as

$$
m=\frac{\left(1-c_{*}^{2}\right) \tilde{m}}{c_{*}^{2}}=\left(c_{*}^{-2}-1\right) \tilde{m} .
$$

\subsection{Gordon form of Lense-Thirring slow-rotation spacetime}

Let us remind ourselves of the quite standard version of the Lense-Thirring slow-rotation spacetime (in the usual Schwarzschild curvature coordinates). The line element is:

$$
\mathrm{d} s^{2}=-\left(1-\frac{2 m}{r}\right) \mathrm{d} t^{2}+\left(1-\frac{2 m}{r}\right)^{-1} \mathrm{~d} r^{2}+r^{2} \mathrm{~d} \theta^{2}+r^{2} \sin ^{2} \theta\left(\mathrm{d} \phi-\frac{2 m a}{r^{3}} \mathrm{~d} t\right)^{2} .
$$

This represents a metric which is Schwarzschild (in curvature coordinates) plus $O(a)$ modifications, and for this metric one can easily check that $R_{a b}=O\left(a^{2}\right)$; all components of the Ricci tensor are $O\left(a^{2}\right)$. This $O\left(a^{2}\right)$ behaviour for the Ricci tensor is what we mean by saying that the Lense-Thirring spacetime is an approximate solution to the vacuum Einstein equations corresponding to a slowly rotating spacetime. The spacetime has angular momentum $J=m a$. For current purposes we could equally well ignore the $O\left(a^{2}\right)$ term in the metric and write the simplified Lense-Thirring line element as:

$\mathrm{d} s^{2}=-\left(1-\frac{2 m}{r}\right) \mathrm{d} t^{2}+\left(1-\frac{2 m}{r}\right)^{-1} \mathrm{~d} r^{2}+r^{2} \mathrm{~d} \theta^{2}+r^{2} \sin ^{2} \theta \mathrm{d} \phi^{2}-\frac{4 m a \sin ^{2} \theta}{r} \mathrm{~d} t \mathrm{~d} \phi$.

This simplified line element represents a metric which is still Schwarzschild (in curvature coordinates) plus $O(a)$ modifications, and for this metric we still get $R_{a b}=O\left(a^{2}\right)$; all components of the Ricci tensor are $O\left(a^{2}\right)$. That is, the spacetime is still Ricci flat up to terms quadratic in $a$.

Based on these observations, to find a Gordon form for Lense-Thirring we simply take the Gordon form of Schwarzschild and make the ansatz

$$
V=V_{\mu} \mathrm{d} x^{\mu} \rightarrow-\sqrt{1+\frac{2 \tilde{m}}{r}} \mathrm{~d} t+\sqrt{\frac{2 \tilde{m}}{r}} \mathrm{~d} r+\frac{2 \tilde{m} \tilde{a} \sin ^{2} \theta}{r \sqrt{1+\frac{2 \tilde{m}}{r}}} \mathrm{~d} \phi .
$$


That is, we consider the metric ansatz represented by the line element

$$
\begin{aligned}
\mathrm{d} s^{2}= & -\mathrm{d} t^{2}+\mathrm{d} r^{2}+r^{2}\left(\mathrm{~d} \theta^{2}+\sin ^{2} \theta \mathrm{d} \phi^{2}\right) \\
& +\left(1-c_{*}^{2}\right)\left(-\sqrt{1+\frac{2 \tilde{m}}{r}} \mathrm{~d} t+\sqrt{\frac{2 \tilde{m}}{r}} \mathrm{~d} r+\frac{2 \tilde{m} \tilde{a} \sin ^{2} \theta}{r \sqrt{1+\frac{2 \tilde{m}}{r}}} \mathrm{~d} \phi\right)^{2} .
\end{aligned}
$$

Here we have again $c_{*} \in(0,1)$, the parameter $\tilde{m}$ is proportional to the physical mass of the Lense-Thirring spacetime, and $\tilde{a}$ is proportional to $a$. Note that (in the background metric) $\|V\|^{2}=-1+O\left(\tilde{a}^{2}\right)$, so that $V$ is approximately a unit timelike 4-vector. Furthermore, since obviously $\tilde{a}=O(a)$, to first order in $a$ this metric ansatz is the just Gordon form of Schwarzschild plus an $O(a)$ perturbation. Finally, a brief computation verifies that $R_{a b}=O\left(a^{2}\right)$, the metric is Ricci-flat to $O\left(a^{2}\right)$. This observation justifies calling this metric the Gordon form of Lense-Thirring spacetime. That is, for slow rotation, we can approximate the Kerr spacetime to arbitrary accuracy by a metric that is of the Gordon form.

To see how the parameters $\tilde{a}$ and $\tilde{m}$ are related to the physical parameters $a$ and $m$, note that at very large $r$ we have $g_{t t} \rightarrow-c_{*}^{2}$, while at all values of $r$ we have $g_{t \phi}=2\left(1-c_{*}^{2}\right) \tilde{m} \tilde{a} \sin ^{2} \theta / r$. Comparing this to the equivalent results for the usual form of the Lense-Thirring line element, (where at very large $r$ we have $g_{t t} \rightarrow-1$, while at all values of $r$ we have $\left.g_{t \phi}=2 m a \sin ^{2} \theta / r\right)$, we see that:

$$
J=m a=\frac{\left(1-c_{*}^{2}\right) \tilde{m} \tilde{a}}{c_{*}}=\frac{\left(1-c_{*}^{2}\right) \tilde{m}}{c_{*}^{2}} \times\left(c_{*} \tilde{a}\right)=m \times\left(c_{*} \tilde{a}\right) .
$$

That is, $a=c_{*} \tilde{a}$, while $m=\left(c_{*}^{-2}-1\right) \tilde{m}$.

\section{General algorithm}

Now let us try to make these observations more systematic by presenting a general algorithm for searching for the Gordon form (if it exists).

\subsection{Non-normalized Gordon and Kerr-Schild forms}

Both Gordon and Kerr-Schild forms of the metric express the metric tensor as the sum of a Riemann-flat background metric $\bar{g}_{\mu \nu}$ and a 1-form $v_{\mu}$ in tensor product with itself. Let us adopt the notation

$$
g_{\mu \nu}=\bar{g}_{\mu \nu}+v_{\mu} v_{\nu}
$$

Here $v_{\mu}$ is not normalized; this lack of normalization is useful in some explicit computations. If $v$ is timelike (with respect to the background metric) then the Gordon form expression of equation (1) can be recovered normalizing $v_{\mu}=\|v\| V_{\mu}$. If $v$ is null then we call this a Kerr-Schild form for the metric tensor. (The remaining case where $v$ is spacelike does not seem to be particularly interesting.) In general, letting $\bar{g}^{\mu \nu}$ denote 
the inverse of the flat background metric, which here we do not necessarily presume has to be in the form $\eta_{\mu \nu}=\operatorname{diag}(-1,+1,+1,+1)$, the inverse of the full metric is

$$
g^{\mu \nu}=\bar{g}^{\mu \nu}-\frac{\bar{g}^{\mu \alpha} \bar{g}^{\nu \beta} v_{\alpha} v_{\beta}}{1+\bar{g}^{\rho \sigma} v_{\rho} v_{\sigma}} .
$$

The specific choice of coordinates is manifestly irrelevant for this description: As long as the metric tensor can be put into a Gordon form, every coordinate transformation that acts on both sides will provide an equivalent expression for the same decomposition. It is in principle possible to find inequivalent Gordon forms for the same spacetime if, choosing a common flat background metric, different 1-forms provide different full metric tensors which are equivalent through coordinate transformations.

\subsection{How to find analytic expressions for Gordon and Kerr-Schild forms}

Knowing an expression for the full metric in a certain set of coordinates $g_{\mu \nu}$, and an expression for the flat metric in a generally different set of coordinates $\bar{g}_{\alpha \beta}$, we look for possible inequivalent Gordon forms of the metric by applying a coordinate transformation of the form

$$
x^{\mu} \rightarrow x^{\prime \mu}=x^{\mu}+\xi^{\mu}(x),
$$

and then noting

$$
\begin{aligned}
g_{\mu \nu}(x) \mathrm{d} x^{\mu} \mathrm{d} x^{\nu} & \rightarrow g_{\mu \nu}\left(x^{\prime}\right) \mathrm{d} x^{\prime \mu} \mathrm{d} x^{\prime \nu} \\
& =g_{\mu \nu}(x+\xi)\left(\delta_{\alpha}^{\mu}+\frac{\partial \xi^{\mu}}{\partial x^{\alpha}}\right)\left(\delta_{\beta}^{\nu}+\frac{\partial \xi^{\nu}}{\partial x^{\beta}}\right) \mathrm{d} x^{\alpha} \mathrm{d} x^{\beta} .
\end{aligned}
$$

The RHS of equation (15) is a new expression for the spacetime metric which depends on the chosen local translations $\xi$ defining the coordinate transformations of equation (14). This expression can be written in a Gordon form if it is possible to find a 1-form $v$ satisfying

$$
g_{\mu \nu}(x+\xi)\left(\delta_{\alpha}^{\mu}+\partial_{\alpha} \xi^{\mu}\right)\left(\delta_{\beta}^{\nu}+\partial_{\beta} \xi^{\nu}\right)-\bar{g}_{\alpha \beta}(x)=v_{\alpha} v_{\beta} .
$$

It is a straightforward algebraic exercise to extract - up to an overall sign - the expressions for the functions $v_{\alpha}$ in terms of the functions $\xi^{\mu}$ and their derivatives, from four of these ten equations. The remaining six equations provide a system of highly nontrivial and non-linear partial differential equations for the functions $\xi^{\mu}$ and the initially chosen tensors $g$ and $\bar{g}$.

Ultimately the problem of finding a Gordon form for the metric is that of finding an appropriate coordinate transformation, i.e., solving the differential equations for the $\xi^{\mu}$, such that the initial system equation (16) admits a solution. In particular, it will be operationally convenient to investigate a class of coordinate transformations which is general enough to find a solution, but possibly without spoiling the explicit symmetries

of the metric. The Schwarzschild spacetime is a remarkable example of a system where this problem admits an explicit solution, and we first use this to present a specific implementation of the general algorithm. 


\subsection{Checking Schwarzschild in Kerr-Schild form}

We first apply this procedure to recover the well known Kerr-Schild form of the Schwarzschild metric, describing a static black hole of physical mass $m$. The usual expression for the metric obviously requires a transformation of coordinates to be put in a Gordon form since when choosing spherical coordinates for the flat background (mildly abusing notation by conflating metrics with their line elements)

$$
\bar{g}_{\text {spherical }}=-\mathrm{d} t^{2}+\mathrm{d} r^{2}+r^{2} \mathrm{~d} \theta^{2}+r^{2} \sin ^{2} \theta \mathrm{d} \phi^{2},
$$

we have

$$
\begin{aligned}
g & =-\left(1-\frac{2 m}{r}\right) \mathrm{d} t^{2}+\left(1-\frac{2 m}{r}\right)^{-1} \mathrm{~d} r^{2}+r^{2} \mathrm{~d} \theta^{2}+r^{2} \sin ^{2} \theta \mathrm{d} \phi^{2} \\
& \neq \bar{g}_{\text {spherical }}+v \otimes v .
\end{aligned}
$$

We need to apply a coordinate transformation which does not spoil the explicit time translation symmetry and the explicit spherical symmetry. The spatial coordinates are better left untouched since the angular part of both the full metric and the spherical flat background is the same: $v$ must have vanishing angular components, making a rotation completely irrelevant; moreover, $r^{2}$ appears as the scale factor of the angular part of both metric tensors, and therefore $r$ cannot be transformed. So we shall initially consider the simple coordinate transformation $t \rightarrow t+f(r)$.

Applying such transformation to the Schwarzschild metric, and choosing as flat background the spherical flat metric $\bar{g}_{\text {spherical, }}$ the system in equation (16) admits a solution for $f^{\prime}(r)=\mp \frac{2 m}{r-2 m}$. That is, we obtain the Kerr-Schild expression for the Schwarzschild spacetime, as equation (12), with

$$
\begin{aligned}
& g=\bar{g}_{\text {spherical }}+v \otimes v, \\
& v=-\sqrt{\frac{2 m}{r}} \mathrm{~d} t \mp \sqrt{\frac{2 m}{r}} \mathrm{~d} r .
\end{aligned}
$$

The 1-form $v$ defines a Kerr-Schild decomposition since it is a null 1-form, as is easily checked by verifying $\bar{g}^{\mu \nu} v_{\mu} v_{\nu}=0$. The overall sign is chosen in such a way that the dual of this 1-form is a future-directed vector field.

In this case the process of solving the system of equations (16) only requires the expression for $f^{\prime}(r)$, which can be obtained algebraically: The analytical expression for $f(r)$ itself is not needed; it is enough to know that $f^{\prime}(r)$ is integrable to be sure that the coordinate transformation is properly defined. The computation was made particularly easy by an appropriate choice of background: The flat metric in spherical coordinates explicitly contains the same symmetries as the full spacetime, and this background is simply the limit of the full initial metric for vanishing black hole mass $m \rightarrow 0$. (This can also be considered as the limiting case of vanishing angular momentum for the known solution of the Kerr-Schild form of the Kerr metric.) 


\subsection{Checking Schwarzschild in Gordon form}

A second application of the general algorithm allows us to recover the known result of the Gordon form of the Schwarzschild metric: by considering a somewhat more general class of coordinate transformations it is possible to find inequivalent non-null 1-forms reproducing the Schwarzschild metric as in the Gordon form of equation (12).

The reasoning presented above suggested that it would be profitable to consider a translation of the $t$ coordinate by a function of the radial coordinate $r$ only, so that the explicit symmetries of the metric were preserved. However, more generally we note that the time translation symmetry is still explicitly preserved if the $t$ coordinate is deformed by rescaling. So a wider class of coordinate transformations to apply to the standard Schwarzschild metric equation (18) is this

$$
t \rightarrow \sqrt{1-\zeta} t+f(r) ; \quad \mathrm{d} t \rightarrow \sqrt{1-\zeta} \mathrm{d} t+f^{\prime}(r) \mathrm{d} r .
$$

We will consider the rescaling factor $\sqrt{1-\zeta}=c_{*}$ many times in the following discussion, and we will usually refer to $\zeta$ as the deformation parameter. This class of coordinate transformations modifies the appearance of the metric tensor, and the metric can be written in Gordon form with respect to the flat spherical background, (that is, a solution for the system of equations (16) with $\bar{g}=\bar{g}_{\text {spherical }}$ exists), if and only if

$$
f^{\prime}(r)=\mp \frac{2 m}{r-2 m} \sqrt{1+\zeta \frac{r-2 m}{2 m}} .
$$

Since $f^{\prime}(r)$ is integrable this describes a proper coordinate transformation.

In conclusion, the Schwarzschild metric can be cast in a Gordon form with

$$
\begin{aligned}
& g=\bar{g}_{\text {spherical }}+v \otimes v, \\
& v=-\sqrt{\zeta+\frac{2 m}{r}(1-\zeta)} \mathrm{d} t \mp \sqrt{\frac{2 m}{r}(1-\zeta)} \mathrm{d} r .
\end{aligned}
$$

The 1-form $v$ is in general non-null, since $\bar{g}^{\mu \nu} v_{\mu} v_{\nu}=-\zeta$; the limit $\zeta \rightarrow 0$ reproduces the Kerr-Schild form. The original expression of the Gordon form of equation (11), or equation (4), is obtained by rewriting these expressions in terms of the speed of light in the medium and the normalized 4-velocity:

$$
\begin{aligned}
c_{*}^{2} & =1-\zeta, \quad \zeta=1-c_{*}^{2}, \\
V & =\frac{v}{\sqrt{\zeta}}=-\sqrt{1+\frac{2 \tilde{m}}{r}} \mathrm{~d} t \mp \sqrt{\frac{2 \tilde{m}}{r}} \mathrm{~d} r, \\
\tilde{m} & =m \frac{1-\zeta}{\zeta} .
\end{aligned}
$$

Here $m$ is again the physical mass, while $\tilde{m}$ is a convenient shorthand. The parameter $\zeta$ is bounded from both sides: In order to transform the $t$ coordinate we must have $\zeta<1$, otherwise we wouldn't be able to consider the square root $\sqrt{1-\zeta}$. From equation (23) we also understand that it must be required that the parameter $\zeta$ be non-negative: In

order for the square root $\sqrt{1+\zeta \frac{r-2 m}{2 m}}$ to exist in the external region $r>2 m$, we must 
have $\zeta \geq 0$. In conclusion, the deformation parameter is bounded within a finite interval $\zeta \in[0,1)$, which corresponds to $c_{*}^{2} \in(0,1]$, the speed of light in the medium should be real, non-negative, and bounded by the speed of light in vacuum.

\section{The Kerr spacetime}

We now consider the more interesting case of rotating black holes, described with the Kerr metric, which we would like to express in Gordon form.

It is well-known that the Kerr metric can be written in Kerr-Schild form, which we could obtain following an analogous procedure to the one seen for the Schwarzschild spacetime. For our purposes, it is most convenient to consider the expression of the metric tensor as presented in Kerr's original derivation [16]. Again slightly abusing notation by conflating the metric with its corresponding line element we have

$$
\begin{aligned}
g= & \left(r^{2}+a^{2} \cos ^{2} \theta\right)\left(\mathrm{d} \theta^{2}+\sin ^{2} \theta \mathrm{d} \phi^{2}\right)+2\left(\mathrm{~d} u+a \sin ^{2} \theta \mathrm{d} \phi\right)\left(\mathrm{d} r+a \sin ^{2} \theta \mathrm{d} \phi\right) \\
& -\left(1-\frac{2 m r}{r^{2}+a^{2} \cos ^{2} \theta}\right)\left(\mathrm{d} u+a \sin ^{2} \theta \mathrm{d} \phi\right)^{2},
\end{aligned}
$$

where $u$ should be read as a modified time coordinate (the advanced/retarded time). Applying the transformation $u \rightarrow \pm t+r$ to this metric we easily obtain the KerrSchild form of the Kerr metric, making use of a non-trivial representation of the flat background

$$
g=\bar{g}_{\text {spheroidal }}+v \otimes v
$$

Here

$\bar{g}_{\text {spheroidal }}=-\mathrm{d} t^{2}+\mathrm{d} r^{2}+2 a \sin ^{2} \theta \mathrm{d} r \mathrm{~d} \phi+\left(r^{2}+a^{2} \cos ^{2} \theta\right) \mathrm{d} \theta^{2}+\left(r^{2}+a^{2}\right) \sin ^{2} \theta \mathrm{d} \phi^{2}$,

is a non-trivial non-diagonal implementation of spheroidal coordinates, and the null 1 -form $v$ is given by

$$
v=\sqrt{\frac{2 m r}{r^{2}+a^{2} \cos ^{2} \theta}}\left(-\mathrm{d} t \mp \mathrm{d} r \mp a \sin ^{2} \theta \mathrm{d} \phi\right) .
$$

As expected, the limit $a \rightarrow 0$ of $\bar{g}_{\text {spheroidal }}$ yields the spherical polar flat metric, while the limit $a \rightarrow 0$ of $v$ reproduces the 1-form of equation (21) for the Kerr-Schild form of the Schwarzschild metric, as presented in equation (201). This decomposition is therefore a general description for Kerr, of which the Schwarzschild version is a particular case. The norm of $v$ can be shown - after computing $\bar{g}_{\text {spheroidal }}^{\mu \nu}$ to be vanishing, proving that this expression is indeed of Kerr-Schild form.

The flat background metric (31) considered here is the limit $m \rightarrow 0$ of the full metric; it is indeed Riemann-flat since it is obtained from the usual spherical flat metric equation (17) through a (somewhat non-obvious) coordinate transformation

$$
r^{2} \rightarrow r^{2}+a^{2} \sin ^{2} \theta
$$




$$
\begin{aligned}
\sin ^{2} \theta & \rightarrow \frac{\left(r^{2}+a^{2}\right) \sin ^{2} \theta}{r^{2}+a^{2} \sin ^{2} \theta}, \\
\phi & \rightarrow \phi+\arctan \left(\frac{r}{a}\right) .
\end{aligned}
$$

Note that not transforming the coordinate $\phi$ as done in equation (35) would have resulted in obtaining the somewhat more usual diagonal form of oblate spheroidal coordinates (without the $\mathrm{d} r \mathrm{~d} \phi$ cross term).

To apply the general procedure described in subsection 3.2 and to search for a Gordon form of the Kerr metric, we now need to manipulate the metric tensor with a sufficiently wide class of coordinate transformations. Indeed, this will be considerably less trivial than for the Schwarzschild metric, simply because the Kerr spacetime has fewer symmetries. This implies that, while previously we could assume transformations preserving spherical symmetry, now in the Kerr spacetime we can only make weaker assumptions, as only an axial symmetry is left in the spatial sector. Expressing the coordinate transformation in terms of local translations as in equation (14), we can expect the translation to depend neither on $\phi$, nor on $t$, apart from again possibly rescaling $t$ by the factor $\sqrt{1-\zeta}=c_{*}$. Accordingly we consider

$$
x^{\mu} \rightarrow x^{\mu}+(\sqrt{1-\zeta}-1) \delta_{t}^{\mu} t+G^{\mu}(r, \theta) .
$$

In general all four coordinates should now be transformed.

As discussed in subsection 3.2, the full resolution of the system of equations (16) requires solving nonlinear (quadratic) partial differential equations. Therefore finding the exact Gordon form for the Kerr metric seems (at least for now) to be a step too far. But we can certainly investigate this system perturbatively: We can expect that for a small rotation parameter $a$ the perturbative expression of the Gordon form of the Kerr spacetime is a perturbation of the Gordon form of the Schwarzschild spacetime. Moreover, for a small deformation of the time coordinate - thereby considering a description at first order in $\zeta$ - we can also expect the Gordon form of the Kerr spacetime to be a perturbation of the known Kerr-Schild form of the Kerr metric.

In the following analysis we present these two different perturbative approaches, and the general expressions resulting from the resolution of the system (16) within the two separate approximations of slow-rotation and a near-null 1-form.

\subsection{Slow rotation}

When considering the slow-rotation regime, we can adopt approximate descriptions of the Kerr metric equivalent to the Lense-Thirring metric (8), which can be interpreted as a perturbation of the Schwarzschild metric. So in this subsection we shall consider a perturbative expansion for small angular momentum: We look for a Gordon form approximating the Kerr metric at first order in $a$. This is a common assumption in the literature, which is physically reasonable since astrophysical rotating black holes

must certainly have $a<m$, (albeit they can become almost extreme due to accretion processes). Initially we discuss how to obtain the full solution of the system of 
equation (16) in the case of the Kerr metric approximated at order $a$; what we obtain is the most general first-order (in $a$ ) approximation to the Gordon form of the Kerr metric. Then we make a consistency check with the Gordon form of the Lense-Thirring metric as expressed in equation (10).

\subsubsection{General case of slow-rotating Kerr in Gordon form}

To obtain the most general Gordon form of the Kerr metric at order $a$, one should proceed as described previously in section 3.2. transforming the first-order approximation of the Kerr metric, (this is simply equations (30)-(31)-(32) with the 1-form $v$ approximated to first order in $a$ )

$$
\bar{g}_{\text {spheroidal }}+\left(-\sqrt{\frac{2 m}{r}} \mathrm{~d} t \mp \sqrt{\frac{2 m}{r}} \mathrm{~d} r \mp a \sqrt{\frac{2 m}{r}} \sin ^{2} \theta \mathrm{d} \phi\right)^{2}+O\left(a^{2}\right),
$$

with the most general coordinate transformation which preserves the explicit axial symmetry and time translation symmetry,

$$
\begin{aligned}
t & \rightarrow \sqrt{1-\zeta} t+\tilde{f}(r)+a G^{t}(r, \theta)+O\left(a^{2}\right), \\
r & \rightarrow r+a G^{r}(r, \theta)+O\left(a^{2}\right), \\
\theta & \rightarrow \theta+\frac{a}{2 m} G^{\theta}(r, \theta)+O\left(a^{2}\right), \\
\phi & \rightarrow \phi+\frac{a}{2 m} G^{\phi}(r, \theta)+O\left(a^{2}\right) .
\end{aligned}
$$

Here the function $\tilde{f}$ in equation (38) must (see equation (23)) have derivative

$$
\tilde{f}^{\prime}(r)= \pm \frac{2 m}{r-2 m}\left(1-\sqrt{1+\zeta \frac{r-2 m}{2 m}}\right),
$$

for consistency with what we already know is needed, in the case of vanishing $a$, to put the Kerr-Schild form of the Schwarzschild geometry into Gordon form.

In order to find the most general Gordon form of the Kerr metric at order $a$, the functions $G^{\mu}$ generating the coordinate transformation must be solutions of the system of equations (16) - after linearization with respect to $a$ - together with an appropriate 1-form $v$. The solution at zeroth order $a^{0}$ is known, since it will simply be the Gordon form of the Schwarzschild metric. At next higher order the 1-form will include a correction of order $a$ for every component. So we will have

$$
v=-\sqrt{\zeta+\frac{2 m}{r}(1-\zeta)} \mathrm{d} t \mp \sqrt{\frac{2 m}{r}(1-\zeta)} \mathrm{d} r+a \delta v+O\left(a^{2}\right) .
$$

Taking the first order approximation, the nonlinear system of equations (16) is reduced to a system of first-order partial differential equations. This system can be

solved patiently, step by step — first obtaining the expressions for the components $\delta v_{\mu}$ in terms of the functions $G^{\mu}$ and their derivatives, and then solving the system, finding their explicit expressions. The integration constants should be fixed in such a way that for vanishing $\zeta$ the 1 -form obtained ultimately reduces to that defining the Kerr-Schild metric (37). 
Here is the full expression obtained for the Gordon form of the Kerr spacetime, written in the form $g=\bar{g}_{\text {spheroidal }}+v \otimes v$, at first order in $a$ :

$$
\begin{aligned}
& v_{t}=-\sqrt{\frac{2 m}{r}\left(1+\zeta \frac{r-2 m}{2 m}\right)} \\
& -\frac{a \kappa}{2 r} \sqrt{\frac{2 m}{r} \frac{(1-\zeta)^{2}}{1+\zeta \frac{r-2 m}{2 m}}}\left(1-\sqrt{1+\zeta \frac{r-2 m}{2 m}}\right) \cos \theta+O\left(a^{2}\right), \\
& v_{r}=\mp \sqrt{\frac{2 m}{r}(1-\zeta)} \\
& \mp \frac{a \kappa}{2 r} \sqrt{\frac{2 m}{r}(1-\zeta)}\left(\left(1-\sqrt{1+\zeta \frac{r-2 m}{2 m}}\right)+\frac{\zeta r^{2}}{4 m^{2} \sqrt{1+\zeta \frac{r-2 m}{2 m}}}\right) \cos \theta \\
& +O\left(a^{2}\right) \\
& v_{\theta}=\mp a \kappa \sqrt{\frac{2 m}{r}(1-\zeta)}\left(\left(1-\sqrt{1+\zeta \frac{r-2 m}{2 m}}\right)-\frac{\zeta r(r-2 m)}{8 m^{2} \sqrt{1+\zeta \frac{r-2 m}{2 m}}}\right) \sin \theta \\
& +O\left(a^{2}\right), \\
& v_{\phi}=\mp a \sqrt{\frac{2 m}{r} \frac{1-\zeta}{1+\zeta \frac{r-2 m}{2 m}}} \sin ^{2} \theta+O\left(a^{2}\right) \text {. }
\end{aligned}
$$

Here $\kappa$ is a dimensionless residual integration constant one finds from the coordinate transformation described by equations (38)-(41), when the integration constants are chosen to be independent both from $a$ and $\zeta$. This Gordon form correctly describes the Kerr spacetime up to order $a^{2}$, i.e. it can be verified that this Gordon form produces a vanishing Ricci tensor up to $O\left(a^{2}\right)$. This was expected since we simply considered coordinate transformations of the Kerr metric; with this check the formalism used is therefore proven to be consistent.

We observe that in general the norm of the 1-form $v$ is non trivial: for non-vanishing $\kappa$, it has a contribution of order $a$ which strongly depends on the angular and radial position

$$
\bar{g}^{\mu \nu} v_{\mu} v_{\nu}=-\zeta\left(1+\frac{a \kappa}{2 m} \frac{1-\zeta}{\sqrt{1+\zeta \frac{r-2 m}{2 m}}} \cos \theta\right)+O\left(a^{2}\right) .
$$

In the limit of null deformation parameter $\zeta \rightarrow 0$, in which case the 1 -form $v$ reproduces the Kerr-Schild case - the norm vanishes identically, for any value of $\kappa$.

That is, the first order in a Gordon form of the Kerr metric has been obtained as a perturbation of the Gordon form of the Schwarzschild metric, (which is the limiting case for vanishing $a$ ). It should be noted that in this more general case the deformation parameter $\zeta$ is again bounded within the same interval, $\zeta \in[0,1)$. If the integration constant $\kappa$ is not neglected the norm is highly point-dependent; in particular it may be possible that the norm changes sign due to the presence of the factor $\cos \theta$ in the term 
of order $a$. To avoid this change of sign, a bound on $\kappa$ should be imposed. To define this bound we make the assumptions $0 \leq \zeta<1$ and $\left|\frac{a}{M}\right|<1$, and we consider as the region of interest that with $r>2 m$ (implying $\left.1<\left(1+\zeta \frac{r-2 m}{2 m}\right)^{1 / 2}<\infty\right)$. With such assumptions we find that $|\kappa|<2$ always prevents a change of sign of the norm of the 1-form. In general, for a given $a / m$ ratio, one needs $|\kappa|<|2 m / a|$.

\subsubsection{Consistency check with Lense-Thirring}

We have found a general expression for the Gordon form of the slow rotating Kerr spacetime. We can now easily make a consistency check to prove that this Gordon form, defined in terms of the spheroidal flat background and the 1-form of components (44)-(47), is equivalent to the Lense-Thirring Gordon form introduced in equations (9) and (10).

This can be done assuming a vanishing integration constant $\kappa$ and making use of the properly redefined parameters — as discussed previously — of the speed of light in the medium and rescaled mass and angular momentum parameters

$$
\begin{aligned}
\kappa & =0, \\
c_{*}^{2} & =1-\zeta+O\left(a^{2}\right), \\
\tilde{m} & =m \frac{1-\zeta}{\zeta}, \\
\tilde{a} & =\frac{a}{\sqrt{1-\zeta}} .
\end{aligned}
$$

Extracting the normalization of the 1 -form $v$ (for vanishing $\kappa$ its norm is simply $\sqrt{\zeta}$ ) and substituting the parameters, we obtain the same expression for $v$ as equation (9)

$$
V=\frac{v}{\sqrt{\zeta}}=-\sqrt{1+\frac{2 \tilde{m}}{r}} \mathrm{~d} t \mp \sqrt{\frac{2 \tilde{m}}{r}} \mathrm{~d} r \mp \frac{2 \tilde{m} \tilde{a} \sin ^{2} \theta}{r \sqrt{1+\frac{2 \tilde{m}}{r}}} \mathrm{~d} \phi+O\left(\tilde{a}^{2}\right) .
$$

Actually, this is not yet quite enough to verify the equivalence between the two Gordon forms because this 1-form is referred to the spheroidal flat background, while the LenseThirring Gordon form in equation (10) is referred to the spherical flat metric. However with a final simple coordinate transformation it is possible to prove that these Gordon forms are completely equivalent: The inverse of the transformation from the spherical flat metric to the spheroidal flat metric - approximated at order $a$, and therefore transforming only the coordinate $\phi$ with the inverse of the transformation (35) - is indeed what is needed, since it properly transforms the flat background and does not modify the 1-form up to order $O\left(a^{2}\right)$. This transformation is

$$
\begin{aligned}
\mathrm{d} \phi & \rightarrow \mathrm{d} \phi-\frac{a}{r^{2}} \mathrm{~d} r+O\left(a^{2}\right), \\
\bar{g}_{\text {spheroidal }} & \rightarrow \bar{g}_{\text {spherical }}+O\left(a^{2}\right),
\end{aligned}
$$

while $V$ (and so $v$ ) is the same. We have therefore proved the equivalence between these two Gordon forms, they can be obtained one from the other through a coordinate transformation. 


\subsection{Near-null Gordon form of Kerr spacetime}

In this section we consider a different approach to the problem of the description of the Kerr metric in a Gordon form. We have already seen that - given a fixed flat background - inequivalent Gordon forms of the metric can be obtained through a class of coordinate transformations which include a rescaling of the time coordinate. To

describe such deformations we have used the rescaling term $\sqrt{1-\zeta}$. Rescaling the time coordinate, one can obtain new 1-forms with non-null norm, passing from a Kerr-Schild form to a Gordon form. The parameter $\zeta$ is therefore strictly related to the norm and becomes the instrument to explore the space of possible inequivalent Gordon forms of the Kerr spacetime.

In the slow-rotation case, the order $a$ approximation has allowed us to consider Gordon forms where the rescaling parameter $\zeta$ was free to explore the whole range of allowed values: That is, the same interval $\zeta \in[0,1)$ which was acceptable in the Schwarzschild case. While the small $a$ result is already of great interest, it rules out the whole regime of rapidly rotating spacetimes. We now wish to explore that region of parameter space, and we want to do so by making a different approximation: We consider a small deformation parameter $\zeta$, and we obtain an expression for the Gordon form of the Kerr metric at first order in $\zeta$.

\subsubsection{Infinitesimal local translation of the Kerr metric}

We want to apply the procedure described in the introductory subsection (3.2), transforming the Kerr-Schild form of the Kerr metric (30) with an infinitesimal transformation. Again we want this coordinate transformation to include a deformation of the time coordinate; since we want this deformation to be infinitesimal, we can approximate at order $\zeta$ the rescaling term, and consider $\zeta$ to be arbitrary small

$$
\sqrt{1-\zeta}=1-\frac{\zeta}{2}+O\left(\zeta^{2}\right) .
$$

The other coordinates should be transformed infinitesimally too, and the rest of the transformation should be assumed not to spoil the explicit axial and time translation symmetries. So we consider

$$
\begin{aligned}
& t \rightarrow\left(1-\frac{\zeta}{2}\right) t+\zeta F^{t}(r, \theta)+O\left(\zeta^{2}\right), \\
& r \rightarrow r+\zeta F^{r}(r, \theta)+O\left(\zeta^{2}\right), \\
& \theta \rightarrow \theta+\zeta F^{\theta}(r, \theta)+O\left(\zeta^{2}\right), \\
& \phi \rightarrow \phi+\zeta F^{\phi}(r, \theta)+O\left(\zeta^{2}\right) .
\end{aligned}
$$

This coordinate transformation is easily brought back to the formalism of infinitesimal local translations as presented in section (3.2). The coordinates are transformed with $x^{\mu} \rightarrow x^{\mu}+\xi^{\mu}(x)$, and the translation vector $\xi$ defining this coordinate transformation is clearly of order $\zeta$. This means that for an arbitrarily small $\zeta$, the Kerr metric (30) is moved infinitesimally along the vector field $\xi$, and for this infinitesimal transformation 
it can be written in terms of the Lie derivative along this vector field

$$
g_{\mu \nu} \rightarrow g_{\mu \nu}+\mathcal{L}_{\xi} g_{\mu \nu}=g_{\mu \nu}+\left(\mathcal{L}_{\xi} \bar{g}_{\mu \nu}+v_{\mu} \mathcal{L}_{\xi} v_{\nu}+\mathcal{L}_{\xi} v_{\mu} v_{\nu}\right)
$$

where we are now dropping the subscript in the flat background metric, writing $\bar{g}$ instead of $\bar{g}_{\text {spheroidal }}$ for simplicity. Now of course the relevant question is: Can the transformed expression of the Kerr metric be written in Gordon form? This will be the focus of the next section.

\subsubsection{Solution in modified components}

The infinitesimal translation transforms the Kerr-Schild form metric (30) into the modified metric tensor (61) through the Lie derivative; both are metric tensors describing the Kerr spacetime with the same set of coordinates. The new expression can be put in a Gordon form if it is possible to find a new 1-form which solves, together with vector field $\xi$, the system of equations (16).

The new 1-form must differ from that defining the initial Kerr-Schild form in equation (32) by a term of order $\zeta$, such that the system admits a solution valid for every small value of the deformation parameter; i.e. if the Kerr-Schild form was given (with respect to the spheroidal flat background) by the 1-form

$$
\begin{aligned}
& v=\Phi\left(-\mathrm{d} t \mp \mathrm{d} r \mp a \sin ^{2} \theta \mathrm{d} \phi\right), \\
& \Phi=\sqrt{\frac{2 m r}{r^{2}+a^{2} \cos ^{2} \theta},}
\end{aligned}
$$

where the function $\Phi$ is a shorthand defined for convenience. Then the new solution must be of the form $v+\delta v$, with $\delta v$ being the correction of order $\zeta$. With this assumption for the modified metric tensor and the 1-form, equation (16) is expressed at order $\zeta$ as

$$
\begin{aligned}
g_{\mu \nu}+\left(\mathcal{L}_{\xi} \bar{g}_{\mu \nu}+v_{\mu} \mathcal{L}_{\xi} v_{\nu}+\mathcal{L}_{\xi} v_{\mu} v_{\nu}\right)-\bar{g}_{\mu \nu}=\left(v_{\mu}+\delta v_{\mu}\right)\left(v_{\nu}+\delta v_{\nu}\right), \\
\Downarrow \\
\mathcal{L}_{\xi} \bar{g}_{\mu \nu}+v_{\mu} \mathcal{L}_{\xi} v_{\nu}+\mathcal{L}_{\xi} v_{\mu} v_{\nu}=v_{\mu} \delta v_{\nu}+\delta v_{\mu} v_{\nu}+O\left(\zeta^{2}\right) .
\end{aligned}
$$

It is easier to solve this problem by change of dependent variables, redefining the 1 -form of interest. If we consider the modified 1-form correction

$$
\delta v^{\prime}=\delta v-\mathcal{L}_{\xi} v,
$$

then equation (16), which we have already reduced to (65), takes the simplified form

$$
\mathcal{L}_{\xi} \bar{g}_{\mu \nu}=v_{\mu} \delta v_{\nu}^{\prime}+\delta v_{\mu}^{\prime} v_{\nu}+O\left(\zeta^{2}\right)
$$

For this system, similarly to what has been done for the general case of the Gordon form in the slowly rotating approximation for Kerr spacetime as presented in section (4.1.1), the solution is found step by step. First we obtain the expressions for the components of $\delta v^{\prime}$ in terms of the components - and their derivatives - of the translation vector field $\xi$. Then the system is solved by sequentially finding the functions $\xi^{\mu}$ one after the other. The integration constants should be chosen such that the coordinate transformation reduces to the identity for vanishing $\zeta$, ensuring that no trivial translations in the 
coordinates $t$ and $\phi$ are introduced. Doing all this, the components of the translation vector field are found (to order $O(\zeta)$ ) to be

$$
\begin{aligned}
\xi^{t} & =\left(-\frac{1}{2} t \mp \frac{r^{2}+a^{2} \cos ^{2} \theta}{2 r}\right) \zeta, \\
\xi^{r} & =\left(\lambda \frac{\left(r^{2}+a^{2}\right) \cos \theta}{r^{2}+a^{2} \cos ^{2} \theta}+\frac{a^{2}}{2}\left(\frac{\cos ^{2} \theta}{r}-\frac{r \sin ^{2} \theta}{r^{2}+a^{2} \cos ^{2} \theta}\right)\right) \zeta, \\
\xi^{\theta} & =\left(-\lambda \frac{r \sin \theta}{r^{2}+a^{2} \cos ^{2} \theta}-\frac{a^{2}}{2} \frac{\sin \theta \cos \theta}{r^{2}+a^{2} \cos ^{2} \theta}\right) \zeta, \\
\xi^{\phi} & =\left(-\lambda \frac{a \cos \theta}{r^{2}+a^{2} \cos ^{2} \theta}+\frac{a}{2} \frac{r}{r^{2}+a^{2} \cos ^{2} \theta}\right) \zeta .
\end{aligned}
$$

Here $\lambda$ is a dimensionful integration constant. Inserting these back into the expressions for the $\delta v^{\prime}$, and evaluating the Lie derivative of the 1-form $v$, we obtain the solutions (to order $O(\zeta)$ ) for the components of the modified 1-form $v+\delta v$ on the spehoridal flat background $\bar{g}=\bar{g}_{\text {spheroidal }}$. We first note

$$
v_{\mu}+\delta v_{\mu}=v_{\mu}+\delta v_{\mu}^{\prime}+\mathcal{L}_{\xi} v_{\mu}=v_{\mu}+\delta v_{\mu}^{\prime}+\xi^{\sigma} \partial_{\sigma} v_{\mu}+v_{\sigma} \partial_{\mu} \xi^{\sigma}
$$

The components of $\delta v^{\prime}$ are easily found by substitution after solving equation (67). We find

$$
\delta v^{\prime}=\frac{\zeta}{2 \Phi}\left(-\mathrm{d} t \pm \frac{a^{2} \cos ^{2} \theta}{r^{2}} \mathrm{~d} r \pm \frac{a^{2} \sin 2 \theta}{r} \mathrm{~d} \theta \pm a \sin ^{2} \theta \mathrm{d} \phi\right)+O\left(\zeta^{2}\right) .
$$

The other contribution to $\delta v$ comes from the Lie derivatives of the 1-form $v$ with respect to the translation vector field $\xi$, and in general depends on the integration constant $\lambda$

$$
\begin{aligned}
\mathcal{L}_{\xi} v= & \left(\mathcal{L}_{\xi} \Phi\right)\left(-\mathrm{d} t \mp \mathrm{d} r \mp a \sin ^{2} \theta \mathrm{d} \phi\right) \\
& +\frac{\Phi}{2}\left(\mathrm{~d} t \pm \mathrm{d} r \pm 2 \frac{a^{2} r \cos \theta \sin \theta}{r^{2}+a^{2} \cos ^{2} \theta} \mathrm{d} \theta \pm 2 \frac{a^{3} \cos ^{2} \theta \sin ^{2} \theta}{r^{2}+a^{2} \cos ^{2} \theta} \mathrm{d} \phi\right) \\
& \pm \lambda \Phi\left(\frac{r^{2}-a^{2} \cos ^{2} \theta}{r^{2}+a^{2} \cos ^{2} \theta} \mathrm{d} \theta+2 \frac{a r \cos \theta \sin \theta}{r^{2}+a^{2} \cos ^{2} \theta} \mathrm{d} \phi\right),
\end{aligned}
$$

where

$$
\begin{aligned}
\mathcal{L}_{\xi} \Phi= & -\Phi \frac{\left(2 r^{4} a^{2} \cos (2 \theta)+3 r^{2} a^{4} \sin (2 \theta)-2 a^{6} \cos ^{6} \theta\right)}{8 r^{2}\left(a^{2} \cos ^{2} \theta+r^{2}\right)^{2}} \\
& -\lambda \Phi \frac{\left(r^{4}+3 r^{2} a^{2} \sin ^{2} \theta-a^{4} \cos ^{2} \theta\right)}{2 r\left(a^{2} \cos ^{2} \theta+r^{2}\right)^{2}} \cos \theta .
\end{aligned}
$$

It is interesting to note that the components $\delta v^{\prime}$ can be interpreted as providing the components of the same 1 -form as $\delta v$, when the chosen background is not $\bar{g}$ but $\bar{g}-\mathcal{L}_{\xi} \bar{g}$. This tensor, differing from the flat background by its Lie derivative is still an acceptable approximately flat background (up to order $\zeta$ in the metric and order $\zeta^{2}$ in the Riemann tensor) in order to put the Kerr metric in a Gordon form. Manipulating the expression of the metric (61) with the solution found from equation (67), we can verify that

$$
g_{\mu \nu}=\left(\bar{g}_{\mu \nu}-\mathcal{L}_{\xi} \bar{g}_{\mu \nu}\right)+\left(v_{\mu}+\delta v_{\mu}^{\prime}\right)\left(v_{\nu}+\delta v_{\nu}^{\prime}\right) .
$$


This means that the Kerr metric in Kerr-Schild form can be manipulated with Lie derivatives with respect to the vector field $\xi$ we obtained, in order to write it down as a Gordon form with a modified flat background and a modified 1-form. It is in this setup that is more convenient to evaluate the norm of the 1-form, which can be evaluated straightforwardly by the known inverse of the metric

$$
g^{\mu \nu}\left(v_{\mu}+\delta v_{\mu}^{\prime}\right)\left(v_{\nu}+\delta v_{\nu}^{\prime}\right)=-\zeta \frac{r^{2}+a^{2} \cos ^{2} \theta}{r^{2}}+O\left(\zeta^{2}\right),
$$

proving that this is really a Gordon form for a near null 1-form.

\section{Vorticity and applications to analogue spacetimes}

Interest in the Gordon form of spacetime metrics is due (among other things) to potential applications in the analogue spacetime programme. See specifically [22, 32], and more generally [28, 29, 30, 31, 33, 34, 35, 36. Indeed the Gordon form, (or something conformal to the Gordon form), generically describes the acoustic metric experienced by a linearised perturbation on a relativistic fluid [22, 32]. That one might want vorticity in analogue systems is clear from references [37, 38, 39, 40, 41, 42, 43, 44. Very often, however, in theoretical analyses of these analogue systems the inclusion of vorticity is tricky [45] - most typically the four velocity of the fluid is by construction hypersurface orthogonal (implying that it can be written as being proportional to the gradient of some scalar function) and as such - by the Frobenius theorem - it is vorticity free (in the relativistic sense that $V \wedge \mathrm{d} V=0$ ). This is potentially a problem for an experimental simulation of a true Kerr geometry given that one can very easily realise that the KerrSchild and Kerr-Gordon forms of the Kerr metric found in this paper always require a four velocity (or equivalently a one-form) which is not vorticity free. This fact can be seen most easily by looking at the simplest case, the four velocity obtained in the Gordon form of the Lense-Thirring spacetime,

$$
V=-\sqrt{1+\frac{2 \tilde{m}}{r}} \mathrm{~d} t \mp \sqrt{\frac{2 \tilde{m}}{r}} \mathrm{~d} r \mp \tilde{a} \sqrt{\frac{2 \tilde{m}}{r} \frac{2 \tilde{m}}{r+2 \tilde{m}}} \sin ^{2} \theta \mathrm{d} \phi+O\left(a^{2}\right) .
$$

For this four velocity we can compute the 4-vorticity

$$
\begin{aligned}
\omega=\star(V \wedge \mathrm{d} V) & =V_{\nu_{1}} V_{\nu_{3}, \nu_{2}} \star\left(\mathrm{d} x^{\nu_{1}} \wedge \mathrm{d} x^{\nu_{2}} \wedge \mathrm{d} x^{\nu_{3}}\right) \\
& =V_{\nu_{1}} V_{\nu_{3}, \nu_{2}} \epsilon^{\nu_{1} \nu_{2} \nu_{3} \nu_{4}} \bar{g}_{\nu_{4} \mu_{4}} \mathrm{~d} x^{\mu_{4}} \\
& =\sqrt{\bar{g}} V_{\nu_{1}} V_{\nu_{3}, \nu_{2}} \bar{g}^{\nu_{1} \mu_{1}} \bar{g}^{\nu_{2} \mu_{2}} \bar{g}^{\nu_{3} \mu_{3}} \hat{\epsilon}_{\mu_{1} \mu_{2} \mu_{3} \mu_{4}} \mathrm{~d} x^{\mu_{4}} \\
& =-\frac{4 \tilde{a} \tilde{m} \cos \theta}{r^{3}} \sqrt{\frac{2 \tilde{m}}{r+2 \tilde{m}}} \mathrm{~d} t \mp \frac{4 \tilde{a} \tilde{m} \cos \theta}{r^{3}} \mathrm{~d} r \mp \frac{2 \tilde{a} \tilde{m} \sin \theta}{r(2 \tilde{m}+r)} \mathrm{d} \theta .
\end{aligned}
$$

The 4-vorticity 1-form $\omega$ is evaluated with respect to the usual flat background. This result is non vanishing at first order in $a$, and is valid for arbitrary deformation parameter $\zeta \in(0,1)$. This is enough to imply that also for the near-null Gordon form it is impossible for the 4-vorticity to vanish. Indeed, the expression of the 4-vorticity of the near null Gordon form will depend on the parameter $a$; at first order, it must be 
consistent with the non-vanishing expression in equation (83). It will therefore also be impossible for the 4-vorticity in the near-null case to vanish identically.

Hence we conclude that any analogue model of the Kerr geometry will have to necessarily include vorticity in the background flow. Models of this sort are indeed available and are discussed in a companion paper [12].

\section{Further developments - towards a full solution}

We have presented two approximate results for the Gordon form of the Kerr metric, describing the regimes of small rotation and near-null 1-form; they were obtained respectively by perturbing the Gordon form of the Schwarzschild metric, and by infinitesimally deforming the Kerr-Schild form of the Kerr metric. These two results plausibly suggest the existence of an (as yet unknown) full analytical expression for the Gordon form of the Kerr metric, one which should reproduce the results presented herein when considering the first order approximations in the two parameters $a$ and $\zeta$. Such an expression would be the full solution of the system of equations (16), possibly found through the algorithm presented in subsection 3.2 .

Finally, we remark that in finding these results we found that a proper choice of flat background is of crucial importance for the resolution of the problem - both in terms of the final expression for the Gordon form, and in computation time, and this should be considered in any future approach to this problem. We hope to further explore these and other possibilities in the next future.

\section{Acknowledgments}

MV was supported by the Marsden Fund, which is administered by the Royal Society of New Zealand. MV would also like to thank both SISSA and INFN (Trieste) for hospitality during the initial phase of this work.

\section{References}

[1] Walter Gordon, "Zur Lichtfortpflanzung nach der Relativitätstheorie", Annalen der Physik 377(22) (1923) 421-456; doi: 10.1002/andp.19233772202 "On the propagation of light in the theory of relativity", translated by D. H. Delphenich. http://www.neo-classical-physics.info/uploads/3/4/3/6/34363841/gordon_-_optical_metrics.pdf

[2] Landau, L.D. and Lifshitz, E.M., The classical theory of fields, (Pergamon Press, Oxford, 1971), 3rd edition.

[3] J. Plebański, "Electromagnetic waves in gravitational fields", Phys. Rev., 118, 1396-1408, (1960).

[4] J. Plebański, Lectures on Nonlinear Electrodynamics, (Nordita, Copenhagen, 1970).

[5] F. de Felice, "On the gravitational field acting as an optical medium", Gen. Relativ. Gravit., 2, 347-357, (1971).

[6] Skrotskii, G.V., "The influence of gravitation on the propagation of light", Sov. Phys. Dokl., 2, 226-229, (1957).

[7] Balazs, N.L., "Effect of a gravitational field, due to a rotating body, on the plane of polarization of an electromagnetic wave", Phys. Rev., 110, 236-239, (1958). 
[8] Anderson, J.L. and Spiegel, E.A., "Radiative transfer through a flowing refractive medium", Astrophys. J., 202, 454-464, (1975).

[9] Pham, Q.M., "Sur les équations de l'electromagné dans la materie", C. R. Hebd. Seanc. Acad. Sci., 242, 465-467, (1956).

[10] S. Schuster and M. Visser, "Effective metrics and a fully covariant description of constitutive tensors in electrodynamics", Physical Review D 96 (2017)124019 doi: 10.1103/PhysRevD.96.124019 arXiv:1706.06280 [gr-qc]].

[11] S. Schuster and M. Visser, "Bespoke analogue space-times: Meta-material mimics", arXiv:1801.05549 [gr-qc].

[12] S. Liberati, S. Schuster, G. Tricella, and M. Visser, "Vorticity in analogue spacetimes", arXiv:1802.04785 [gr-qc].

[13] S. Schuster and M. Visser, "Boyer-Lindquist space-times and beyond: Meta-material analogues," arXiv:1802.09807 [gr-qc].

[14] K. Rosquist, "A Moving medium simulation of Schwarzschild black hole optics", Gen. Rel. Grav. 36 (2004) 1977 doi: 10.1023/B:GERG.0000036055.82140.06 gr-qc/0309104.

[15] L. Giacomelli and S. Liberati, "Rotating black hole solutions in relativistic analogue gravity", Phys. Rev. D 96 (2017) no.6, 064014 doi:10.1103/PhysRevD.96.064014 arXiv:1705.05696 [gr-qc]].

[16] R. P. Kerr, "Gravitational field of a spinning mass as an example of algebraically special metrics", Phys. Rev. Lett. 11 (1963) 237. doi:10.1103/PhysRevLett.11.237

[17] D. L. Wiltshire, M. Visser and S. M. Scott (editors), "The Kerr spacetime: Rotating black holes in general relativity", (Cambridge University Press, Cambridge, England, 2009)

[18] M. Visser, "The Kerr spacetime: A brief introduction", arXiv:0706.0622 [gr-qc]. Published in [17.

[19] S. A. Teukolsky, "The Kerr metric", Class. Quant. Grav. 32 (2015) no.12, 124006 doi:10.1088/02649381/32/12/124006 arXiv:1410.2130 [gr-qc]].

[20] C. Doran, "A new form of the Kerr solution", Phys. Rev. D 61 (2000) 067503 doi:10.1103/PhysRevD.61.067503 gr-qc/9910099.

[21] A. J. S. Hamilton and J. P. Lisle, "The river model of black holes", Am. J. Phys. 76 (2008) 519 doi:10.1119/1.2830526 gr-qc/0411060.

[22] M. Visser and C. Molina-París,

"Acoustic geometry for general relativistic barotropic irrotational fluid flow",

New J. Phys. 12 (2010) 095014 doi:10.1088/1367-2630/12/9/095014 arXiv:1001.1310 [gr-qc]].

[23] W. G. Unruh, "Experimental black hole evaporation", Phys. Rev. Lett. 46 (1981) 1351. doi:10.1103/PhysRevLett.46.1351

[24] M. Visser, "Acoustic propagation in fluids: An unexpected example of Lorentzian geometry", gr-qc/9311028.

[25] M. Visser, "Acoustic black holes: Horizons, ergospheres, and Hawking radiation", Class. Quant. Grav. 15 (1998) 1767 doi:10.1088/0264-9381/15/6/024 gr-qc/9712010.

[26] C. Barceló, S. Liberati and M. Visser, "Analog gravity from Bose-Einstein condensates", Class. Quant. Grav. 18 (2001) 1137 doi:10.1088/0264-9381/18/6/312 gr-qc/0011026.

[27] C. Barceló, S. Liberati and M. Visser, "Probing semiclassical analog gravity in Bose-Einstein condensates with widely tunable interactions", Phys. Rev. A 68 (2003) 053613 doi:10.1103/PhysRevA.68.053613 cond-mat/0307491.

[28] C. Barceló, S. Liberati and M. Visser, "Analogue gravity", Living Rev. Rel. 8 (2005) 12 [Living Rev. Rel. 14 (2011) 3] doi: 10.12942/lrr-2005-12 gr-qc/0505065].

[29] M. Visser, C. Barceló and S. Liberati, "Analog models of and for gravity", Gen. Rel. Grav. 34 (2002) 1719 doi:10.1023/A:1020180409214 gr-qc/0111111.

[30] M. Visser and S. Weinfurtner, "Analogue spacetimes: Toy models for "quantum gravity"”, PoS QG -PH (2007) 042 arXiv:0712.0427 [gr-qc]]. 
[31] M. Visser, "Survey of analogue spacetimes", Lect. Notes Phys. 870 (2013) 31 doi:10.1007/978-3-319-00266-8_2 arXiv:1206.2397 [gr-qc]].

[32] S. Fagnocchi, S. Finazzi, S. Liberati, M. Kormos and A. Trombettoni, "Relativistic Bose-Einstein condensates: a new system for analogue models of gravity", New J. Phys. 12 (2010) 095012 doi:10.1088/1367-2630/12/9/095012 arXiv:1001.1044 [gr-qc]].

[33] M. Visser and S. Weinfurtner, "Massive phonon modes from a BEC-based analog model", cond-mat/0409639.

[34] M. Visser and S. Weinfurtner,

"Massive Klein-Gordon equation from a BEC-based analogue spacetime", Phys. Rev. D 72 (2005) 044020 doi:10.1103/PhysRevD.72.044020 gr-qc/0506029.

[35] A. Finke, P. Jain and S. Weinfurtner, "On the observation of nonclassical excitations in Bose-Einstein condensates", New J. Phys. 18 (2016) no.11, 113017 doi:10.1088/1367-2630/18/11/113017 arXiv:1601.06766 [quant-ph]].

[36] J. Braden, M. C. Johnson, H. V. Peiris and S. Weinfurtner, "Towards the cold atom analog false vacuum", arXiv:1712.02356 [hep-th].

[37] M. Visser and S. Weinfurtner, "Vortex geometry for the equatorial slice of the Kerr black hole", Class. Quant. Grav. 22 (2005) 2493 doi:10.1088/0264-9381/22/12/011 gr-qc/0409014.

[38] M. Richartz, A. Prain, S. Liberati and S. Weinfurtner, "Rotating black holes in a draining bathtub: super-radiant scattering of gravity waves", Phys. Rev. D 91 (2015) no.12, 124018 doi:10.1103/PhysRevD.91.124018 arXiv:1411.1662 [gr-qc]].

[39] V. Cardoso, A. Coutant, M. Richartz and S. Weinfurtner, "Detecting Rotational Super-radiance in Fluid Laboratories", Phys. Rev. Lett. 117 (2016) no.27, 271101 doi:10.1103/PhysRevLett.117.271101 [arXiv:1607.01378 [gr-qc]].

[40] T. Torres, S. Patrick, A. Coutant, M. Richartz, E. W. Tedford and S. Weinfurtner, "Observation of super-radiance in a vortex flow", Nature Phys. 13 (2017) 833 doi:10.1038/nphys4151 arXiv:1612.06180 [gr-qc]].

[41] T. Torres, A. Coutant, S. Dolan and S. Weinfurtner, "Waves on a vortex: rays, rings and resonances", arXiv:1712.04675 [gr-qc].

[42] S. Patrick, A. Coutant, M. Richartz and S. Weinfurtner, "Black hole quasi-bound states from a draining bathtub vortex flow", arXiv:1801.08473 [gr-qc].

[43] B. Cropp, S. Liberati and R. Turcati, "Vorticity in analog gravity", Class. Quant. Grav. 33 (2016) no.12, 125009 doi:10.1088/0264-9381/33/12/125009 [arXiv:1512.08198 [gr-qc]].

[44] L. Giacomelli and S. Liberati, "Rotating black hole solutions in relativistic analogue gravity", Phys. Rev. D 96 (2017) no.6, 064014 doi:10.1103/PhysRevD.96.064014 arXiv:1705.05696 [gr-qc]].

[45] S. E. Perez Bergliaffa, K. Hibberd, M. Stone and M. Visser, "Wave equation for sound in fluids with vorticity", Physica D 191 (2004) 121 doi:10.1016/j.physd.2003.11.007 cond-mat/0106255]. 